\title{
Thai Agriculture Products Traceability System using Blockchain and Internet of Things
}

\author{
Thattapon Surasak ${ }^{1 *}$, Nungnit Wattanavichean ${ }^{2}$, Chakkrit Preuksakarn ${ }^{3}$, Scott C.-H. Huang ${ }^{4}$ \\ Institute of Communications Engineering, National Tsing Hua University, Hsinchu City, Taiwan ${ }^{1,4}$ \\ Department of Applied Chemistry, National Chiao Tung University, Hsinchu City, Taiwan ${ }^{2}$ \\ Department of Computer Engineering, Kasetsart University, Nakhon Pathom, Thailand ${ }^{3}$
}

\begin{abstract}
In this paper, we successfully designed and developed Thai agriculture products traceability system using blockchain and Internet of Things. Blockchain, which is the distributed database, is used for our proposed traceability system to enhance the transparency and data integrity. OurSQL is added on another layer to easier query process of blockchain database, therefore the proposed system is a user-friendly system, which cannot be found in ordinary blockchain database. The website and android application have been developed to show the tracking information of the product. The blockchain database coupling with Internet of Things give a number of benefits for our traceability system because all of the collecting information is in real-time and kept in a very secured database. Our system could have a huge impact on food traceability and supply chain management become more reliable as well as rebuild public awareness in Thailand on food safety and quality control.
\end{abstract}

Keywords-Blockchain; internet of things; supply chain management; product traceability; distributed database; data integrity; ourSQL

\section{INTRODUCTION}

The Kingdom of Thailand is situated in Southeast Asia. Thailand is an agricultural country. According to The Trading Economics website reported that the Gross Domestic Product (GDP) from agriculture is the major section that raise the overall GDP in 2018. Although there is the rapid expansion of industrial sectors, agriculture is still a majority [1].

However, there are a number of problems and concerns regarding the quality of Thai agricultural products. Not only due to the plant diseases and chemical contamination, which is the main problems, but also the uncontrollable factor such as weather conditions or disasters. These kind of problems cause the reduction of agricultural products quality. Eventually, this problem leads to the lower income of farmer as well as consumer confidence for their receiving products. [1], [2].

The traceability system is an appropriate solution to control, prevent and exterminate various problems and concerns in supply chain, especially in food and agricultural sector [3]. In this process the details of the product since it was farming until delivered to the consumers has been controlled to meet required food safety and quality standards (GMP, HACCP, and HALAL). European Union (EU) has launched a law and policy for the meat products registration and identification to guarantee that the meats products sold in European countries are verifiable and traceable [4]. The traceability system is reliable when it consists of secured database and trusted monitoring system. Both components can be provided by technology [5].
In this paper, blockchain database is implemented with IoT devices.

Blockchain is a new technology that catch many attentions in various fields of researches at this moment. The structure of blockchain illustrates in Fig. 1. It is a chain of blocks which each block stores all information of network activities after the block was added to the chain [6]. This feature makes blockchain become traceable database. Blockchain allows every user to add data as a transaction. Moreover, all data in the blockchain can be reviewed by every user, but no one able to change it [7]. The security and validity of the information in blockchain controlled by one process called 'mining process' [8]. This process aims to verify the information before adding any block to the chain. A person who verifies the new transactions and create the block known as miners. Miners utilize a consensus algorithm to add the new block. This algorithm is similar to a voting system. Once the transaction is confirmed by enough number of nodes (voter), it will be validated and permanently be a part of the database. After the block was added to the database, it cannot be changed. Therefore, this method ensures the transparency, trust, and traceability in a system. As this reason, blockchain is very famous among financial applications. The most well-known is Bitcoin, which is a peer-to-peer electronic cash system [9]. In addition, blockchain has one interesting feature called smart contract, which is the digital agreement. This feature is suitable for supply chain management because it can create a deal between farmers and consumers [7].

Internet of things (IoT) is enabling the connection between machine to machine (M2M) over the Internet [10]. With IoT, sensors, actuators and electronic devices can collect and exchange the data with each others [11]. In food supply chain traceability system, the IoT devices is calibrated to make sure that its measurement value is accurate. The data collected from the IoT device is reported in real-time [12]. One advantage of using IoT device to collect the data is because it reading value cannot manipulate by human [13]. With the implementation to blockchain database, all true value is kept directly in the database without changing, which leads to high reliable of the system [14], [15].

In this paper, we propose a promising solution in which Internet of Things, blockchain distributed database have been integrated. Section II reviews the works related with tracability system by applying the SQL database, blockchain, and IoT technology. Section III explains our proposed blockchain traceability system which consists of the blockchain database technology (OurSQL) and system architecture. Section IV presents 
A

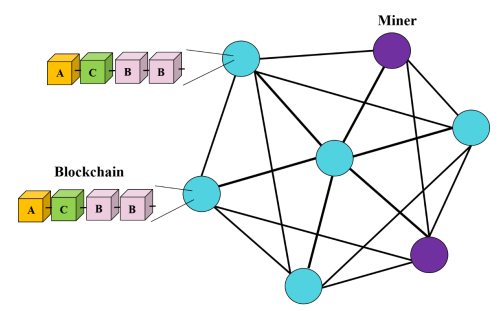

B

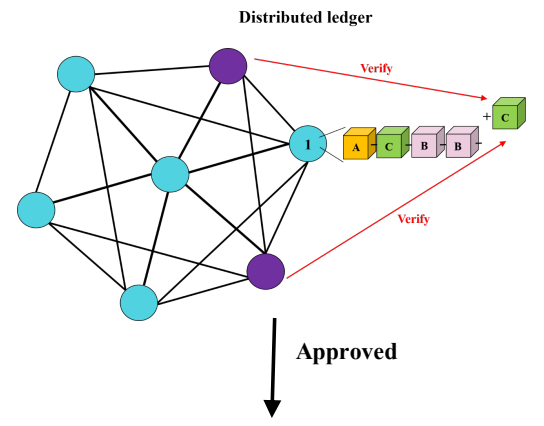

C

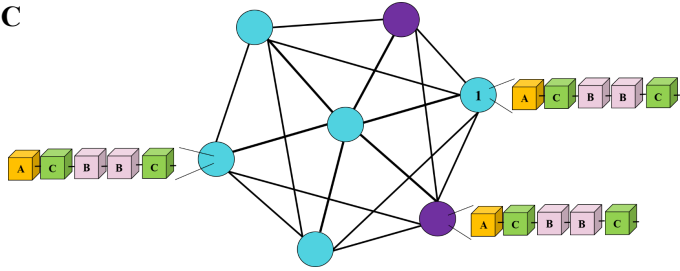

Fig. 1. Blockchain and the block creation process: (A) blockchain and distributed ledger. (B) The block creation process starts when one node (user) requests to make a transaction over the whole blockchain network.

The particular transaction needs to be verified by the others. After transaction verified, the miner can create the block and connect it to the chain. (C) After the block creation process is approved, every node in the system is also updated with a new block created from part (B).

the case study to verify the feasibility of proposed system. The results are shown on website and android application. The evaluation and benchmark of our proposed system design have been discussed. Finally, Section V describes the conclusion and future work.

\section{RELATED WORK}

In traceability system for food and agricultural supply chain, users are able to track and trace the products from the origin [16]. In the past, only database technology was required to create a traceability system and the user could directly put all information into the database. The most popular database to store the product information is SQL database [17]. Although this solution can track the product information, the human error is still another big issue in this data collection process [13].

The use of Internet of Things (IoT) technology introduces many benefits for both business and human aspects [18]. The supply chain management has various advantages by integrating the IoT [19], [20]. For example, the user will be able to use the real-time monitoring system, which is the most important system in the food supply chain, by implementing the IoT traceability system with the main food supply chain logistics [21]. This provides a number of benefits regarding the food quality and security. RFID and QR-code also be implemented in order to allow the users to easily retrieve the information [6], [20], [22]. For the previous work,
ZigBee mesh network has applied to work on the real time GPS tracking and monitoring. This increases the number of benefits in the logistic management for agricultural products. However, there have some problems regarding security issues due to the server misusing [23]. In order to control the temperature and humidity, the wireless sensor networks have been applied. The application can automatically determine whether it going to water the tomato greenhouse. With the system, the tomato greenhouse is in controlled [24]. Furthermore, one of smart farm research in Thailand also used the wireless sensor networks to detect the environmental data of the farm using several sensors. Air temperature, humidity, water temperature and $\mathrm{pH}$ have been chosen as the parameters that represent the quality of planting process. After the data collected, the data were analyzed, summarized, and store in the database, respectively. This information includes name of plants, origins, and farm certification. To monitor the smart farm conditions, an Android application was created. The $\mathrm{QR}$ code also be generated to allow the customers to access additional information after the products have been shipped to the stores. The main advantage of a smart farm and traceability system using IoT, which proposed in this research, let the customer to have more confidence in the agricultural product [25]. Anyway, the more benefits provided, the more emerging security issues about the IoT server and devices [26]. In previous year, an authentication mechanism has been created to protect the system [10]. However, an authentication itself can avoid the misuse problem only because the data could be changed after the users authenticated themselves to the system [27]. Therefore, data integrity is another important part that developer need to carefully designed along with creating the IoT system [11].

The implementation of blockchain with the IoT will be the most strongest system that can applied for the food supply chain [28]-[31]. Recently, the researchers have impressively adapt the blockchain technology with IoT for the supply chain traceability system [31]. One of these applied the blockchain and IoT with soybean traceability [32]. This work creates the smart contract which is one of the blockchain feature to use as a real agreement between seed company and the farmer. The contract is used when the farmer wants to ship their product to distributors or retailers. With this work, the researchers can guarantee that the farmer gets the most benefit they deserve. Blockchain technology has also be adapted with the RFID to use as a food supply chain tracking system in China [6]. The blockchain technology provides the secure database for the food supply chain and the RFID is applied as an interactive database that can be used by scanning the RFID and getting interested information. A research applied the Ethereum blockchain, which is well-known blockchain technology, for traceability system [33]. In order to show the data from Ethereum blockchain, web3 API is used [29]. However, the data stored in ledger can be seen by any user in the Ethereum network which is not suitable for sensitive data. Moreover, using a large-scale network dramatically consumes a lot of time and cost for block creation process. Another shortage of using blockchain as the database is because it required a specific command based on each blockchain technology to query the information [34], [35]. In other word, the common blockchain database is not easy to query the information we need. This problem can be solved by implementation of the 
SQL database with the blockchain technology [7], [36].

\section{BLOCKCHAIN-BASED TRACEABILITY SYSTEM}

As mentioned before, each blockchain technology requires a specific command to retrieve the information. This problem can be solved by implementation of the SQL database with the blockchain technology. In this research, the IoT sensors and OurSQL (Blockchain replication database technology) have been combined together to immediately store the agricultural product information into the blockchain database. In this study, OurSQL is used as a core of the system in order to collect the data from the IoT sensors. OurSQL platform provides a unique ability to share selected information including location tracking, temperature, humidity and ownership transfers. The first implementation of our system provides for the beef products in Ratchaburi Province of Thailand. There are three important information to collect for the beef which are temperature, humidity and location. Therefore, the temperature, humidity and GPS sensors were used to collect the data on our system. In our design, the product information is collected across the whole supply chain. Therefore, the data collection has been divided into three parts. The first part is the information from the cow farm. The second part collected during the beef production. Then, products shipping is the last part of our collection. These three parts will collect the data in the blockchain database by sensors. From the basic concept of the system, the implementation process was designed based on the supply chain system of the beef production as can see in Fig. 2. The last step of our system design is to create website to allow the customers or restaurant owners who bought the beef to track the beef information. With our website, we show the location tracking information with the temperature and humidity of the beef during the shipping process. Moreover, the information of the particular cow is presented to confirm the quality of the whole beef production.

\section{A. OurSQL}

OurSQL is a server software running between a mysql server and software for the Database client. It has been created by Roman Gelembjuk. OurSQL enables the use of basic SQL client tools and libraries to perform distributed ledger as a SQL database. This tool has two core components, including the blockchain management server and database proxy server. Each node operates with a single database of mysql. Blockchain data are kept together with data tables in the same database. After creating a decentralized database and starting blockchain or joining an existing decentralised database on initial, OurSQL node server can be created. A node server listens on two ports: (1) A local mysql client database proxy server, (2) A port to interact with other existing database cluster OurSQL nodes. mysql client connects on a known port to a proxy. This method is the same as connecting directly on "localhost" to mysql server, just specific another port number. With this decentralized database, mysql client can be a SQL application or a DApp. Moreover, the user can be able to perform any sort of SQL query using SQL command with this decentralized database as well. OurSQL system architecture can be seen in Fig. 3 .

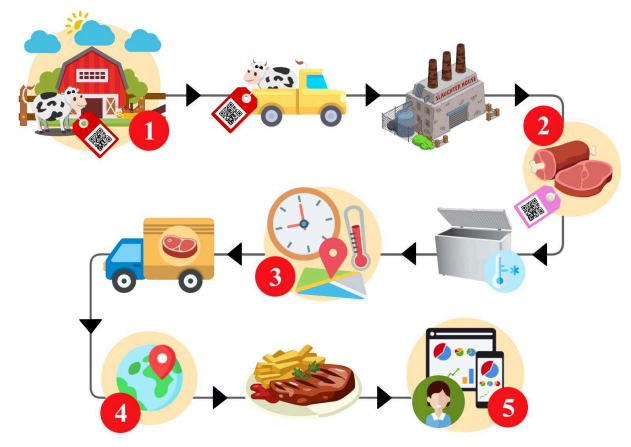

Fig. 2. Data collection methods: (1) The assigned government officer will create a tag (tracking ID and QR code) for a cow and will also track the feeding information during the cow production. When the cow is ready to be slaughtered, the IoT sensors will be used in order to track the cow information during the shipping and transport. (2) After the beef production is finished, the new tag will be allocated with the relation to the previous tag from step (1). (3) and (4) The information will continue track from while the beef has been shipped or delivered from the warehouse. (5) The customers will be able to see the tracking data using the QR code and website.

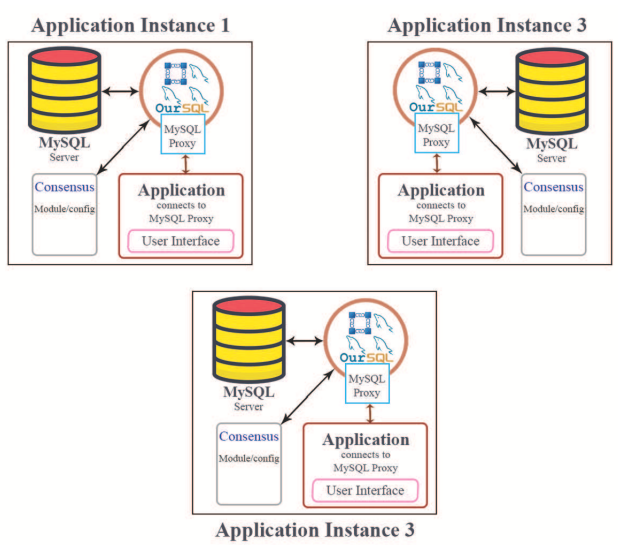

Fig. 3. OurSQL system architecture: It is a no GUI standalone server working on top of mysql server. The key function of OurSQL is to immediately share the updated information when there is an update in the database using Proof of Work (PoW) consensus protocol.

\section{B. System Architecture}

In the aspect of hardware-oriented design, there are three main sections including IoT devices, server, and smart phone. they are connected together via the Internet. IoT devices sends the location with humidity and temperature to the Raspberry $\mathrm{Pi}$. Then the Raspberry Pi forwards the data to the server. If any problem occurs, the actuator system will notify the user using LED and buzzer. Server install the blockchain database (OurSQL) and automatically receive the data from Raspberry Pi. Mobile application is used to control and monitoring the system. Our system design can be seen in Fig. 4(A). For the service-oriented design, this paper divides the system in to three main parts, including client service, system management service, and cloud service can be seen in Fig. 4(B).

1) Client service: The client service can be divides in to two parts: farmer (seller) and general user. Farmer is an user who can add the product lists and information. Moreover, farmer can query the list of the product and also track the 
A

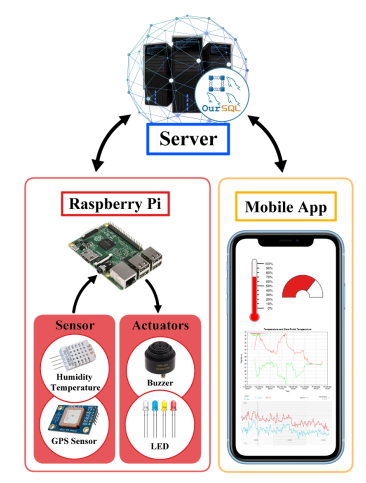

B

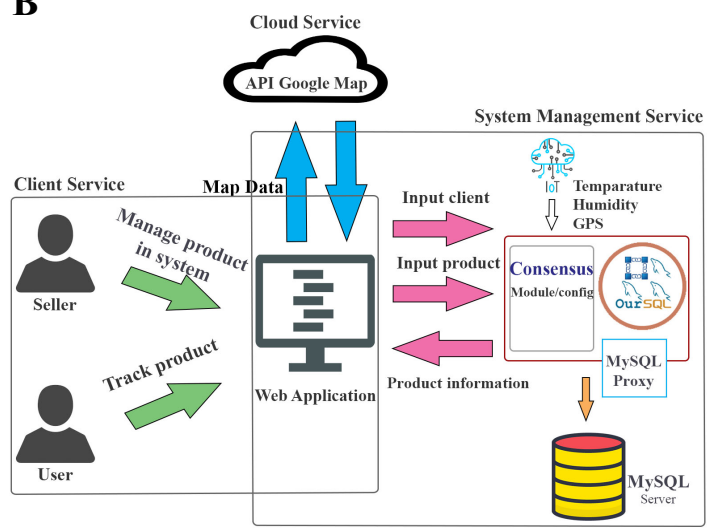

Fig. 4. System design and Overview: In the hardware aspect, this system consists of three main parts: Server, IoT device and Smartphone Application (A). For the service aspect, client service, system management service, and cloud service are three main services need to be separated from each others (B).

product during the shipping process. On the other hand, general user can only see the public information about the product.

2) System management service: It is the control part to manage the website and send/receive the data between the blockchain database and IoT devices.

3) Cloud service: In order to show the real-time tracking system, the Google map API has been used to indicate the coordinate in the map. With this API feature, we are able to track the shipping route in real-time as well as know the specific location of the truck.

\section{RESUlts AND Discussion}

\section{A. Website and Android Application}

After tracking processes finished, our design system automatically generates the QR code for the customer to let the users see the product information. For example, the customer is able to know where the beef come from and when it was first cultivated. With this information, the seller can confirm the quality of the product. We are now successfully created the website and developed the android application in order to show the real-time product information, including location, temperature and humidity.

The website shows the product information from the blockchain database. The location has automatically pinned on the Google map by using the API. Humidity and temperature can be shown during the whole distribution process. The website can be seen in Fig. 5. An android application shows the dash board including virtual temperature and humidity gauges. The status of the system is shown as an alarm on/off. In an ordinary case, the status is shown as an alarm off. However, when a problem is detected, the system status is changed to alarm on. The alarm status and dashboard on the android application can be seen in Fig. 6 .

\section{B. Blockchain Enabled Traceability System}

1) Security of the Database: As mentioned in Section II, data collection methods by the IoT have been applied for a number of studies in order to solve the human errors. However, the current traceability system without the blockchain

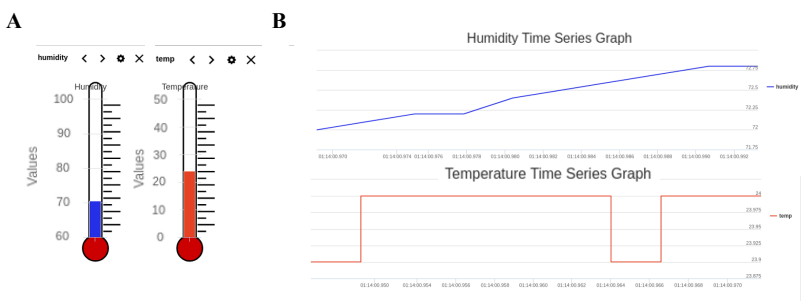

Fig. 5. Website illustrates the humidity and temperature and showed as virtual gauge (A) and time series graph (B).

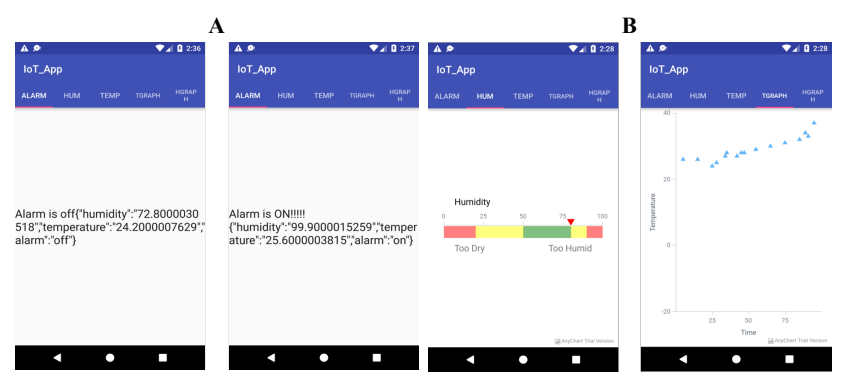

Fig. 6. Android application has two main features: alarm status (A) and time series graph and virtual gauge $(\mathrm{B})$.

integration cannot guarantee the data integrity because hackers still can change the data in the database [26]. There are many attempts which provide authentication procedure to increase data integrity, but these procedures might not be an appropriate solution to these issues and concerns [10], [26]. In the ordinary authentication procedure, it works to avoid the authentication created by the hackers. Unfortunately, the data can also be changed by the users who able to authenticate themselves to the system [10].

So, the traceability systems with the blockchain integration have recently proposed by many researchers. The blockchain technology will increase the data integrity collected by the IoT devices [28]-[31]. The most popular blockchain technology is the Ethereum, which is a public blockchain. Nevertheless, it automatically allows any user in its network to see the data 
stored in ledger. As a result, the sensitive data cannot be stored in this kind of the database. Additionally, using a large-scale network dramatically consumes a lot of time and cost for block creation process.

The implementation of OurSQL, which is a blockchain database, confirm the information security of our system by using the proof of work algorithm in order to create the transactions and the blocks. Therefore, our proposed methods can work with the sensitive data with reasonable time consuming and no block creation fees is needed. This is because OurSQL is a controllable database technology that will allow only selected users to use the system and users do not need to pay for the block creation fees.

2) Query layer for the data stored in the blockchain: In order to store the blockchain to hard disk, the data is required to be compressed. This is meant that these valuable data sets are extremely hard to be reused or shown to the users [36]. Moreover, the normal blockchain database is not easy to query the information because some specific commands based on each blockchain technology are required to query the information [34], [35]. This problem can be solved by implementation of the SQL database with the blockchain technology [7], [36]. In this work, we integrated OurSQL, an efficiency blockchain database, with the IoT data collection method in order to implement the traceability system and show the real-time information via both website and android application. With our proposed database, the website and application development can be finished with the use of SQL commands while using a very secured blockchain replication database as mentioned in subsection III-A.

\section{CONCLUSIONS AND FUTURE WORK}

In this paper, we have integrated the OurSQL with the IoT real-time data collection. To compare with the traditional system (without the blockchain integration), the use of OurSQL blockchain provides unchangeable data when the data stored into the database. In the aspect of blockchain traceability system, our proposed system can utilize the use of SQL commands in the website and application development part. Moreover, OurSQL, controllable blockchain database can work with the sensitive data with a faster query time and no block creation fees is needed. These are our significant improvement points against the traditional methods both with and without the blockchain integration. Our system can check the temperature and humidity of the product in real-time by using website or android application. Moreover, the users can get the notifications when our system found some problems related to the temperature and humidity values. In the future, we plan to add more sensors in order to get more information to ensure the quality of the products. To use all blockchain features, future research can further integrating the traceability system with Hyperledger blockchain technology, which is a permissioned blockchain. This implementation should continue using the SQL database in order to allow the user to use SQL commands with permissioned blockchain. In this permissioned blockchain, the control layer runs on top of the blockchain can differentiate the actions that performed by each user. Therefore, permissioned blockchain has a better transaction performance because we can set the block size limitation and the validate information by adjusting the Chaincode during implementation process. Within this type of blockchain, we can assure the security level of the system because every user has the different priority status. In addition, the smart contract is also recommended in order to avoid the middlemen problem. The deploying of smart contract can be used over many production steps. For example, we can use the smart contract to set up the price and the product quality for both farmers and consumers. Therefore, the farmers are forced to produce their products as good as they can to reach the standard and get the price as mentioned in the contract.

\section{ACKNOWLEDGMENT}

We would like to thank Roman Gelembjuk for the patient guidance and advice regarding OurSQL integration he has provided throughout this project development.

\section{REFERENCES}

[1] N. Poapongsakorn, M. Ruhs, and S. Tangjitwisuth, "Problems and outlook of agriculture in thailand," Thailand Development Research Institute Quarterly Review, vol. 13, 011998.

[2] N. Chomchalow, Agricultural development in Thailand. Dordrecht: Springer Netherlands, 1993, pp. 427-443.

[3] L. U. Opara, "Traceability in agriculture and food supply chain: A review of basic concepts, technological implications, and future prospects," in Food Agricultural and Environment, 2003, pp. 101-106.

[4] S. Ammendrup and L. Barcos, "The implementation of traceability systems," Revue scientifique et technique (International Office of Epizootics), vol. 25, pp. 763-73, 092006.

[5] N. V. Vafiadis and T. T. Taefi, "Differentiating blockchain technology to optimize the processes quality in industry 4.0," in 2019 IEEE 5th World Forum on Internet of Things (WF-IoT), April 2019, pp. 864-869.

[6] F. X. Tian, "An agri-food supply chain traceability system for china based on rfid and blockchain technology," 2016 13th International Conference on Service Systems and Service Management (ICSSSM), pp. 1-6, 2016.

[7] M. Muzammal, Q. Qu, and B. Nasrulin, "Renovating blockchain with distributed databases: An open source system," Future Generation Computer Systems, vol. 90, pp. 105-117, 2019.

[8] Feng, Tian, "A supply chain traceability system for food safety based on haccp, blockchain and internet of things," in 2017 International Conference on Service Systems and Service Management, June 2017, pp. $1-6$.

[9] S. A. Swamy and N. Jayapandian, "Secure bitcoin transaction and iot device usage in decentralized application," in 2018 3rd International Conference on Communication and Electronics Systems (ICCES), Oct 2018, pp. 271-274.

[10] J. Lin, W. Yu, N. Zhang, X. Yang, H. Zhang, and W. Zhao, "A survey on internet of things: Architecture, enabling technologies, security and privacy, and applications," IEEE Internet of Things Journal, vol. 4, no. 5, pp. 1125-1142, Oct 2017.

[11] Y. Yang, L. Wu, G. Yin, L. Li, and H. Zhao, "A survey on security and privacy issues in internet-of-things," IEEE Internet of Things Journal, vol. 4, no. 5, pp. 1250-1258, Oct 2017.

[12] W. Hong, Y. Cai, Z. Yu, and X. Yu, "An agri-product traceability system based on iot and blockchain technology," in 2018 1st IEEE International Conference on Hot Information-Centric Networking (HotICN), Aug 2018, pp. 254-255.

[13] B. Bordel Sánchez, R. Alcarria, D. Martín, and T. Robles, "Tf4sm: A framework for developing traceability solutions in small manufacturing companies," Sensors, vol. 15, pp. 29478-29510, 112015.

[14] S. Rahmadika, B. J. Kweka, C. N. Z. Latt, and K. Rhee, "A preliminary approach of blockchain technology in supply chain system," in 2018 IEEE International Conference on Data Mining Workshops (ICDMW), Nov 2018, pp. 156-160. 
[15] S. Aich, S. Chakraborty, M. Sain, H. Lee, and H. Kim, "A review on benefits of iot integrated blockchain based supply chain management implementations across different sectors with case study," in 2019 21 st International Conference on Advanced Communication Technology (ICACT), Feb 2019, pp. 138-141.

[16] T. M. Bhatt and J. Zhang, "Food product tracing technology capabilities and interoperability." Journal of food science, vol. 78 Suppl 2, pp. B2833, 2013.

[17] B. Adam, R. B. Holcomb, M. Buserc, B. Mayfieldd, J. Thomase, C. A. O’Bryanf, P. Crandallg, D. K. R. Knipei, and S. C. Ricke, "Enhancing food safety, product quality, and value-added in food supply chains using whole-chain traceability," in International Food and Agribusiness Management Review, 2016.

[18] H. Hejazi, H. Rajab, T. Cinkler, and L. Lengyel, "Survey of platforms for massive iot," in 2018 IEEE International Conference on Future IoT Technologies (Future IoT), Jan 2018, pp. 1-8.

[19] L. B. Campos and C. E. Cugnasca, "Towards an iot-based architecture for wine traceability," in 2015 International Conference on Distributed Computing in Sensor Systems, June 2015, pp. 212-213.

[20] W. Liang, J. Cao, Y. Fan, K. Zhu, and Q. Dai, "Modeling and implementation of cattle/beef supply chain traceability using a distributed rfid-based framework in china," PloS one, vol. 10, p. e0139558, 10 2015.

[21] K. Wongpatikaseree, P. Kanka, and A. Ratikan, "Developing smart farm and traceability system for agricultural products using iot technology," in 2018 IEEE/ACIS 17th International Conference on Computer and Information Science (ICIS), June 2018, pp. 180-184.

[22] W. Cao, L. Zheng, H. Zhu, and P. Wu, "General framework for animal food safety traceability using gs1 and rfid," in CCTA, 2009.

[23] G. Angel and A. Brindha, "Real-time monitoring of gps-tracking multifunctional vehicle path control and data acquisition based on zigbee multi-hop mesh network," in 2011 International Conference on Recent Advancements in Electrical, Electronics and Control Engineering, 2011, pp. 398-400.

[24] M. U. H. A. Rasyid, E. M. Kusumaningtyas, and F. Setiawan, "Application to determine water volume for agriculture based on temperature amp; humidity using wireless sensor network," in 2016 International Conference on Knowledge Creation and Intelligent Computing (KCIC), 2016, pp. 105-112.

[25] K. Wongpatikaseree, P. Kanka, and A. Ratikan, "Developing smart farm and traceability system for agricultural products using iot technology," in 2018 IEEE/ACIS 17th International Conference on Computer and Information Science (ICIS), June 2018, pp. 180-184.

[26] A. H. Ngu, M. Gutierrez, V. Metsis, S. Nepal, and Q. Z. Sheng, "Iot middleware: A survey on issues and enabling technologies," IEEE Internet of Things Journal, vol. 4, no. 1, pp. 1-20, Feb 2017.

[27] S. Thattapon and H. Scott C.-H., "Enhancing voip security and efficiency using vpn," in 2019 International Conference on Computing, Networking and Communications (ICNC), Feb 2019, pp. 180-184.

[28] M. Samaniego and R. Deters, "Blockchain as a service for iot," in 2016 IEEE International Conference on Internet of Things (iThings) and IEEE Green Computing and Communications (GreenCom) and IEEE Cyber, Physical and Social Computing (CPSCom) and IEEE Smart Data (SmartData), 2016, pp. 433-436.

[29] M. Kim, B. Hilton, Z. Burks, and J. Reyes, "Integrating blockchain, smart contract-tokens, and iot to design a food traceability solution," in 2018 IEEE 9th Annual Information Technology, Electronics and Mobile Communication Conference (IEMCON), Nov 2018, pp. 335-340.

[30] M. Singh, A. Singh, and S. Kim, "Blockchain: A game changer for securing iot data," in 2018 IEEE 4th World Forum on Internet of Things (WF-IoT), 2018, pp. 51-55.

[31] A. Reyna, C. Martín, J. Chen, E. Soler, and M. Díaz, "On blockchain and its integration with iot. challenges and opportunities," Future Generation Computer Systems, vol. 88, pp. 173-190, 2018.

[32] K. Salah, N. Nizamuddin, R. Jayaraman, and M. Omar, "Blockchainbased soybean traceability in agricultural supply chain," IEEE Access, vol. 7, pp. 73 295-73 305, 2019.

[33] M. P. Caro, M. S. Ali, M. Vecchio, and R. Giaffreda, "Blockchain-based traceability in agri-food supply chain management: A practical implementation," in 2018 IoT Vertical and Topical Summit on Agriculture Tuscany (IOT Tuscany), May 2018, pp. 1-4.

[34] R. Adams, G. Parry, P. Godsiff, and P. Ward, "The future of money and further applications of the blockchain," Strategic Change, vol. 26, no. 5, pp. 417-422, 2017.

[35] E. Karafiloski and A. Mishev, "Blockchain solutions for big data challenges: A literature review," in IEEE EUROCON 2017 -17th International Conference on Smart Technologies, July 2017, pp. 763-768.

[36] Y. Li, K. Zheng, Y. Yan, Q. Liu, and X. Zhou, "Etherql: A query layer for blockchain system," in Database Systems for Advanced Applications, S. Candan, L. Chen, T. B. Pedersen, L. Chang, and W. Hua, Eds. Cham: Springer International Publishing, 2017, pp. 556-567. 